\title{
PRESSURE STUDY OF CHARGED DONOR ORDERING IN HgSe DOPED WITH IRON AND GALLIUM
}

\author{
C. SkierbiszeWski, T. SUSKI \\ High Pressure Research Center, Polish Academy of Sciences \\ Sokolowska 29/37, 01-142 Warszawa, Poland
}

\begin{abstract}
J. Kossut, Z. Wilamowski, W. Dobrowolski and B. Witkowska
Institute of Physics, Polish Academy of Sciences

Al. Lotników 32/46, 02-668 Warszawa, Poland
\end{abstract}

\begin{abstract}
Transport experiments (Hall effect and conductivity) under hydrostatic pressure up to $1 \mathrm{GPa}$ at liquid helium temperatures on $\mathrm{HgSe}: \mathrm{Fe}, \mathrm{Ga}\left(N_{\mathrm{Fe}}=\right.$ $2 \times 10^{19} \mathrm{~cm}^{-3} ; 0 \leq N_{\mathrm{Ga}} \leq 10^{19} \mathrm{~cm}^{-3}$ ) were performed. The results show that the gallium co-doping of HgSe:Fe decreases the degree of spatial correlations between charged impurities. Under the hydrostatic pressure, used as a tool for changing the ratio of the charged to neutral impurities, this effect is even more pronounced. A qualitative agreement between the calculation within the short-range correlation model and our experimental data is achieved.
\end{abstract}

PACS numbers: 72.10.Fk, 72.20.Fr, 72.80.Jc

The pinning of Fermi level to the resonant iron level $E_{\mathrm{Fe}}$ limits the maximum concentration of mobile electrons in zero gap HgSe:Fe. For doping levels higher than $N^{*}=4.5 \times 10^{18} \mathrm{~cm}^{-3}$, the iron impurities exist in their neutral and ionized charge states and in low temperatures, due to Coulomb interactions, the system can lower its energy by redistribution of electrons between charged and neutral $\mathrm{Fe}$ impurities. This leads to a spatial ordering of the ionized donors $[1,2]$. The ordering of charged impurities manifests itself in two ways: (a) as a drastic reduction of the scattering rate by ionized impurities and (b) by changing the Fermi energy which, in turn, influences the concentration of mobile electrons. The ratio between the charged and neutral iron impurities $\left(N_{\mathrm{Fe}+} / N_{\mathrm{Fe}}\right)$ - which can be varied by means of hydrostatic pressure (HP) [3] - is decisive in determining the degree of the spatial ordering of the donor sharges [4]. In this work we investigate $\mathrm{HgSe:Fe}$ samples where apart from the resonant Fe donors, also certain concentrations of other 
type of donors, namely $\mathrm{Ga}$, were intentionally introduced. Since gallium donors in HgSe (at the doping levels used) do not capture electrons in their localized states, therefore co-doping of $\mathrm{HgSe}: \mathrm{Fe}$ with $\mathrm{Ga}$ can be used to modify the ratio $N_{\mathrm{Fe}}+N_{\mathrm{Fe}}$ (the concentration of introduced $\mathrm{Ga}$ is equal to the decrease in the concentration of the de-ionized $\mathrm{Fe}^{+}$). HP can be used with the same purpose thus offering the additional tool for investigation the electron concentration and electron mobility behavior related to the inter-donor correlations with different degree of ordering within one sample. The systematic study of the Hall effect and conductivity at $\mathrm{LHe}^{4}$ temperatures in the pressure range up to $1 \mathrm{GPa}$ was carried out. Five samples with iron concentration $N_{\mathrm{Fe}}=2 \times 10^{19} \mathrm{~cm}^{-3}$ and gallium concentrations $N_{\mathrm{Ga}}=0,1.8,3.6,4.4,10.0\left(\times 10^{18} \mathrm{~cm}^{-3}\right)$ were investigated.

Figure 1 presents. the electron concentration $N_{\mathrm{e}}$ and the mobility $\mu$ as function of gallium concentration. The relative dependences of $N_{\mathrm{e}}(p) / N_{\mathrm{e}}(p=0)$ and

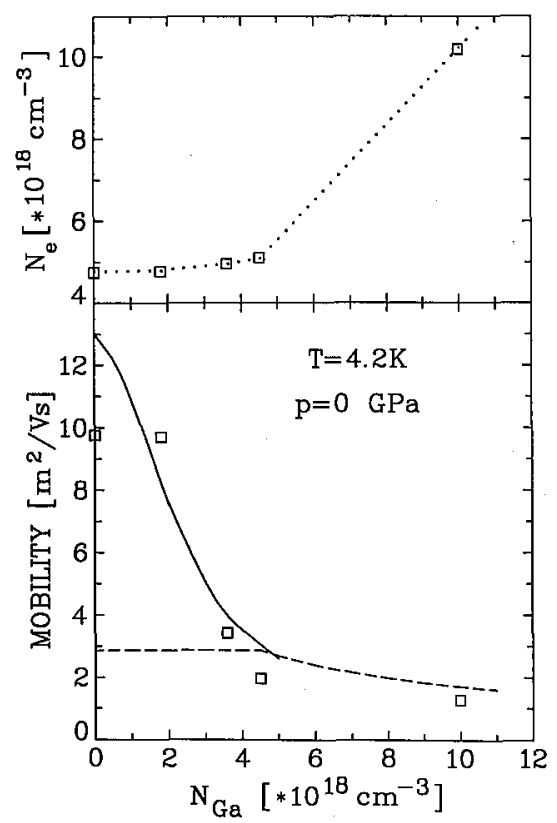

Fig. 1. Electron concentration and mobility as a function of gallium concentration in HgSe: $\mathrm{Fe}, \mathrm{Ga}$ for $N_{\mathrm{Fe}}=2 \times 10^{19} \mathrm{~cm}^{-3}$. Solid line represents calculations within the short-range correlation model (with a constant correlation radius $r_{\mathrm{c}}$ ), while dashed line shows the results of theory for randomly distributed donors. The dotted line is only to guide the eye.

$\mu(p) / \mu(p=0)$ for different $N_{\mathrm{Ga}}$ as a function of applied HP are plotted in Fig. 2. The decrease of the mobility with increasing concentration of gallium confirms the importance of the inter-donor Coulomb interactions in $\mathrm{HgSe}: \mathrm{Fe}, \mathrm{Ga}$ system (where the number of the scatterers and the conduction electron effective mass remain almost constant!). This effect is more pronounced under HP for 


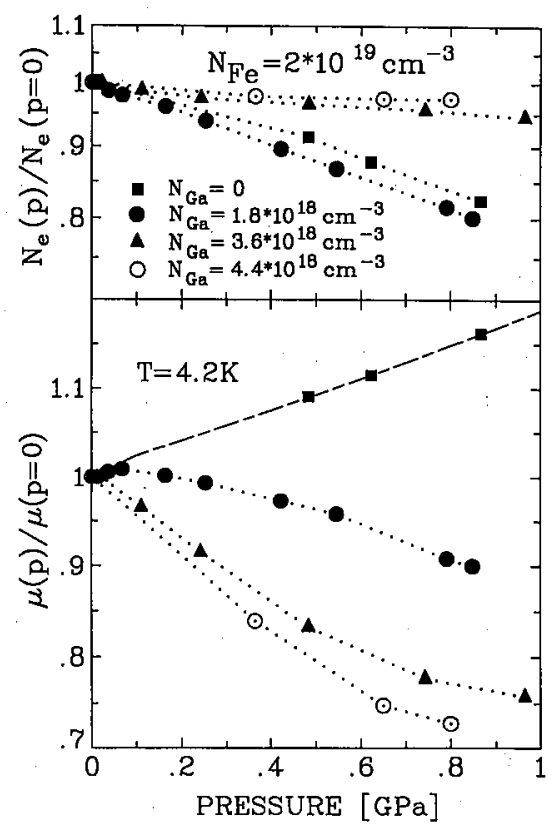

Fig. 2. Relative electron concentration and mobility versus hydrostatic pressure for different doping level of gallium and $N_{\mathrm{Fe}}=2 \times 10^{19} \mathrm{~cm}^{-3}$. The dashed line presents results of calculations of theory for randomly distributed donors (which are almost insensitive to $N_{\mathrm{Ga}}$ ). Dotted lines are only to guide the eye.

$N_{\mathrm{Ga}} \geq 1.8 \times 10^{18} \mathrm{~cm}^{-3}$ where the mobility drop is observed in spite of a decrease of the number of charged scatterers and a decrease of the effective mass.

In the system with two types of charged impurities $\mathrm{Fe}^{+}$and $\mathrm{Ga}^{+}$(denoted as $\alpha$ and $\beta$ ) the following step-like pair correlation functions for $\mathrm{Fe}^{+}-\mathrm{Fe}^{+}$and $\mathrm{Fe}^{+}-\mathrm{Ga}^{+}$donor pairs can be introduced:

$$
g_{\alpha \alpha}(r)=g_{\alpha \beta}(r)=g_{\beta \alpha}(r)=g^{\operatorname{corr}}(r)=\left\{\begin{array}{lll}
0 & \text { for } \quad r<r_{\mathrm{c}} \\
1 & \text { for } \quad r>r_{\mathrm{c}}
\end{array}\right.
$$

where correlation radius $r_{\mathrm{c}}$ has to be independently determined. Between $\mathrm{Ga}^{+}-\mathrm{Ga}^{+}$ donors no correlation occurs, so the corresponding function is defined as $g_{\beta \beta}(r)=$ $g^{\text {random }}(r) \equiv 1$. The total correlation function to be used in the calculation of the momentum relaxation time can be written as:

$$
g^{++}(r)=\left(1-x^{2}\right) \cdot g^{\operatorname{corr}}(r)+x^{2} \cdot g^{\text {random }}(r)
$$

where $x=\left(N_{\mathrm{Ga}^{+}} / N\right) ; N=N_{\mathrm{Ga}^{+}}+N_{\mathrm{Fe}+}$ - the total number of charged impurities. The above formula simply expresses the fact that, for a given total number of charged donors, only a fraction of them can be correlated while the rest (i.e., gallium donors) remains uncorrelated. Thus, by increasing the number of gallium 
donors one lowers the degree of ordering in the system. In order to give the qualitative explanation of the mobility behavior as a function of the gallium doping level, as the first step, the calculations within short range correlation model [4] were performed with a constant value of $r_{\mathrm{c}}=38 \AA\left(r_{\mathrm{c}}\right.$ was obtained for $N_{\mathrm{Fe}}=2 \times 10^{19} \mathrm{~cm}^{-3}$ using suitable equations from [4]). The results show (solid line in Fig. 1) that the main influence on the mobility originates from the changes of $x$ in the function $g^{++}(r)$. For comparison, the mobility calculations for randomly distributed charges [5] are presented as the dashed lines in Fig. 1 and Fig. 2 (the pressure dependences of the relative mobility in this case are almost insensitive to the gallium concentration). Of course, a more detailed analysis of our data is required. In particular, the changes of $r_{\mathrm{c}}$ related to the presence of great number of co-doping gallium ions have to be properly considered.

\section{References}

[1] For review of properties of HgSe:Fe, see: A. Mycielski, J. Appl. Phys. 63, 3279 (1988).

[2] J. Mycielski, Solid State Commun. 60, 165 (1986).

[3] C. Skierbiszewski, T. Suski, E. Litwin-Staszewska, W. Dobrowolski, K. Dybko, A. Mycielski, Semicond. Sci. Technol. 4, 293 (1989).

[4] Z. Wilamowski, K. Świątek, T. Dietl, J. Kossut, Solid State Commun. 74, 833 (1990).

[5] W. Szymańska, T. Dietl, J. Phys. Chem. Solids 39, 1025 (1978). 